\title{
N92-22381
}

\section{RADIATION-INDUCED INSULATOR DISCHARGE PULSES IN THE CRRES INTERNAL DISCHARGE MONITOR SATELLITE EXPERIMENT}

\author{
A. R. Frederickson, E. G. Mullen, \\ D. H. Brautigam and K. J. Kerns, \\ Space Physics Division, \\ Air Force Phillips Laboratory, Hanscom AFB, MA 01731 \\ E. G. Holman, Boston College, Chestnut Hill, MA.
}

\begin{abstract}
The Internal Discharge Monitor, IDM, was designed to observe electrical pulses from common electrical insulators in space service. The sixteen insulator samples include G10 circuit boards, FR4 and PTFE fiberglass circuit boards, wires with common insulations, FEP teflon, and alumina. The samples are fully enclosed, mutually isolated, and space radiation penetrates $0.02 \mathrm{~cm}$ of aluminum before striking the samples. Pulsing began on the seventh orbit, the maximum pulse rate occurred on the seventeenth orbit when 13 pulses occurred, and the pulses slowly diminished to about one per 3 orbits six months later. After 8 months, the radiation belts abruptly increased and the pulse rates attained a new high. These pulse rates are in agreement with laboratory experience on shorter time scales. Several of the samples have never pulsed. If the pulses were not confined within IDM, the physical processes could spread to become a full spacecraft anomaly. The IDM results indicate the rate at which small insulator pulses occur. Small pulses are the seeds of larger satellite electrical anomalies. The pulse rates are compared with space radiation intensities, L shell location, and spectral distribu'ions from the radiation spectrometers on CRRES.
\end{abstract}

\section{INTRODUCTION}

The purpose and background of the Internal Discharge Monitor (IDM) is documented in reference 1 . Nearly twenty years ago it was determined that satellites were suffering from electrical discharge noise and EMP problems caused by the high energy electron space radiation belts. A number of conference sessions, symposia, and journal issues have been dedicated to the topic (Air force, NASA, IEEE, AIAA, ESA). Most of the previous work concentrated on the voltages of surfaces on the satellites. It became clear that the estimated surface voltages were not sufficient to cause the large number of events seen on satellites.

Space radiations acting directly on insulating materials should be able to produce electric pulses, independent of the potential of the spacecraft. The radiation intensity in space is so low that the pulse rates could not be predicted with any certainty. Ground testing, extrapolated to space radiation intensities, hinted that pulses might be as high as a few per day in space [1]. However, the extrapolation is very uncertain because the effects of electrical conductivity at high fields in the insulators can not be predicted in space service. The IDM is measuring the pulses produced by simple insulator structures which are isolated from the effect of the spacecraft potential itself. The IDM results are teaching us that simple insulators, irradiated by space electrons with energies predominantly above 100 $\mathrm{keV}$, produce significant numbers of pulses. The results are also indicating that surface voltage may not be a prime cause of pulses.

The IDM instrument was designed and constructed by the Jet Propulsion Laboratory at the request of the U. S. Air Force. Briefly, the IDM exists to determine if spontaneous electrostatic discharges occur on insulators internal to spacecraft caused by 
penetrating radiation. The IDM has been partially described in a previous publication [1] which reviews test results for IDM sample material under laboratory simulations of the Chemical Release and Radiation Effects Satellite (CRRES) space radiation environment. These test results provided valuable data for determining the final design of the instrument. This paper describes the IDM samples and some results from the first 100 days in space.

The samples were chosen for their generic nature; they are representative of the many materials and device structures used in most spacecraft. In addition, two samples are installed to measure in real time the possible response of the circuit boards of the MicroElectronics Package (MEP) which is adjacent to the IDM on the CRRES satellite.

As radiation (mostly electrons) penetrates the skin of a satellite and stops in the insulating materials, high voltages build up both on and in the insulator materials. Occasional spontaneous discharges of the insulators induce electrical pulses in sensitive satellite wiring. The magnitude of the pulses depends on many parameters including the area of charged insulator surface, the radiation spectrum, existing bias levels on adjacent circuits, the relative area of the sensitive circuit, the effective impedance of the sensitive circuit (as a function of frequency), voltages on other adjacent charged insulator surfaces, a complicated transfer function between the discharging circuit and the sensitive circuit, etc. The total process is much too complex for the IDM to study. The IDM only detects the number of pulses which occur above a threshold level on simple representative generic circuit elements. Thus, it tells us how often such pulses are occurring but nothing about the nature of the pulses. Based on present knowledge (ref. 1 and unpublished personal experience), the IDM is expected to experience pulse rates somewhere between zero and a few per day. The IDM results will significantly improve our knowledge of pulse rates in space.

The IDM is nearly a cubic foot in size, weighs 33 pounds, and uses 6.7 watts continuously. It was designed to last a minimum of two years in its environment. The CRRES orbit is roughly geosynchronous transfer with an apogee of 33,500 $\mathrm{km}$, a perigee of $348 \mathrm{~km}$, a period of 9 hours 52 minutes, and an equatorial inclination of 18.2 degrees. Thus the IDM is exposed to the inner belt for a short time, and to the outer electron belt for a long time. High energy electrons $(>150 \mathrm{keV})$ will be the dominant cause of IDM pulses.

\section{SAMPLES}

The samples were chosen after an extensive testing program as described in reference 1. Table I lists the samples and their configurations as flown on the IDM. The configurations are shown in figure 1 . Most of the samples and configurations are also described in reference 1 , however, configuration $\# 1$ is herein corrected ( $\# 1$ in ref. 1 is not used in IDM) and configurations 7 and 8 are new.

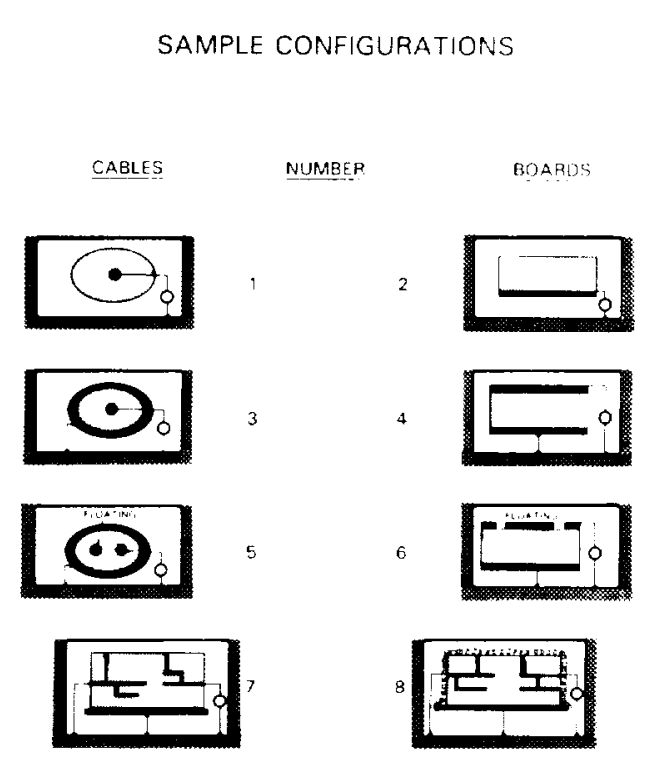

Figure 1. Sample Wiring Configurations. Heavy lines are electrodes, small circles represent the 50 ohm measuring circuit, and the curly line represents leaky paint. Each sample is faraday shielded from external fields by $0.2 \mathrm{~mm}$ aluminum on the top and $0.5 \mathrm{~cm}$ aluminum on the sides and bottom of its container. The feed-through [1] to the external detectors is composed of semi-insulating material which does not pulse. It is not shown in order to simplify the drawing. 


\section{TABLE I. DESCRIPTION OF IDM SAMPLES}

$\mathrm{V}$ max is the maximum pulse voltage during ground tests, CONFIG is the number in figure 1 corresponding to the geometry of electrodes and sample, and PULSES is the number of pulses accumulated in the first one hundred days in space.

\begin{tabular}{||c|l|l|l|c||}
\hline \hline $\begin{array}{l}\text { CHANN } \\
\text { EL }\end{array}$ & SAMPLE DESCRIPTION & $V \mathrm{max}$ & $\begin{array}{l}\text { CONFI } \\
\mathrm{G}\end{array}$ & $\begin{array}{l}\text { PULSE } \\
\mathrm{S}\end{array}$ \\
\hline 1 & SC18 WIRE TYPE ET & 1 & 1 & 9 \\
\hline 2 & TS TRIAX CABLE & 5 & 5 & 0 \\
\hline 3 & $\begin{array}{l}\text { MEP G10 SOLITHANE COATED } \\
\text { ONLY }\end{array}$ & 50 & 7 & 0 \\
\hline 4 & FR4 FIBERGLASS, 0.317 cm & 5 & 2 & 41 \\
\hline 5 & RG 316 CABLE & 0.5 & 3 & 0 \\
\hline 6 & ALJAC CABLE & 1 & 3 & 0 \\
\hline 7 & ALUMINA, 0.102 cm & 40 & 6 & 0 \\
\hline 8 & FR4 FIBERGLASS, 0.317 cm & 1 & 4 & 0 \\
\hline 9 & FEP TEFLON, 0.229 cm & 100 & 6 & 1 \\
\hline 10 & FEP TEFLON, 0.229 cm & 0.2 & 4 & 0 \\
\hline 11 & PTFE FIBERGLASS, 0.229 cm & 1 & 4 & 0 \\
\hline 12 & FR4 FIBERGLASS, 0.317 cm & 5 & 2 & 12 \\
\hline 13 & FR4 FIBERGLASS, 0.317 cm & 100 & 6 & 0 \\
\hline 14 & MEP GI0 SOLITHANE WITH & $<1$ & 8 & 0 \\
\hline 15 & FR4 FIBERGLASS, 0.119 cm & 0.25 & 2 & 39 \\
\hline 16 & PTFE FIBERGLASS, $0.229 \mathrm{~cm}$ & 0.2 & 2 & 163 \\
\hline
\end{tabular}

The maximum pulse voltage measured on a $50 \mathrm{ohm}$ line (sometimes $25 \mathrm{ohms}$ ) is reported in Table I from previous tests: from reference 1 for configurations 16 , and from reference 2 for configurations 7,8 . Because the pulse voltage is so dependent upon many external factors, it is not the exact voltage which is of interest, it is the occurrence of a pulse of sufficient energy to interrupt normal circuit signals that is of interest. Thus, even pulses of order 1 volt on 50 ohms could be of significance for a different geometry or for a circuit with a different transfer function. In different configurations the pulsed voltage, or energy, from the same kind of material could be much larger, especially where the vacuum space surrounding the sample contains high electric fields $(>100 \mathrm{~V} / \mathrm{cm})$ from any source.
Configurations 7 and 8 need a brief discription. Channel 3 contains a sample of MEP circuit board made of G10 material. This is a nine layer board without any mounted components. Approximately one quarter of the circuit board traces are connected to the pulse detector, the rest of the traces are connected to the instrument ground. The surface of the board is treated with urethane (solithane, $\mathrm{tm}$ ) conformal coating. A ground plane is conductively glued to most of the back side of the board. Under $200 \mathrm{keV}$ to $1.4 \mathrm{MeV}$ electron beam testing, similar circuits were found to produce 20 to 40 volt peak pulses several nanoseconds wide [2]. Channel 14 contains a similar sample of MEP board, but it is covered with "leaky paint" [2]. Samples with leaky paint were seen to pulse only rarely and with pulses less than 1 volt in ground tests [2]. 
The leaky paint is an electrically grounded surface coating of semi-insulator with a surface resistivity of the order $10^{10}$ ohms per square. This level of conductivity prevents surface charging but will not electrically short or load the adjacent circuits.

Signal generators (square pulse) were used to produce known pulsed voltage levels at the sample electrodes and thereby determine the thresholds for pulse detection in each channel. In space on the IDM samples, the pulses are expected to vary in width from less than $1 \mathrm{~ns}$ to as much as $20 \mathrm{~ns}$ [1]. The pulse detector thresholds vary depending on pulse width and sensitivity setting. Broadly speaking, the thresholds vary from a tenth of a volt to ten volts on the $50 \mathrm{ohm}$ detectors. Much larger pulses would result from the same phenomena occurring on larger samples or on samples with applied voltage on the electrodes [3,4]. The pulses monitored on these samples are assumed to reflect the phenomena of satellite anomalies produced by discharges on wiring, solar array insulators, antenna insulators, circuit boards, feed-thru insulators, and other electrical component insulation.

The size (total energy) of pulses scales linearly with the area of the dielectric which is charged by the radiation [3]. Since the size of IDM samples is small relative to most spacecraft applications, the IDM instrument was set to be sensitive to small pulsed voltages.

\section{CORRELATION OF PULSES WITH SPACE RADIATION}

The radiation detectors on CRRES provide a good measurement of the radiation spectra which impinge on the IDM. The spectra of electrons incident on the samples should be calculated by transporting the incident CRRES spectra through the $0.20 \mathrm{~mm}$ aluminum cover sheet. Pulse rates would then be correlated with the spectra which directly impact the samples. Since the cover sheet stops all electrons below $150 \mathrm{keV}$, and passes unimpeded the isotropic electron flux above $1 \mathrm{MeV}$, the effect of the cover sheet can be ignored for electrons above $1 \mathrm{MeV}$. The effect on electrons from $150 \mathrm{keV}$ to $1 \mathrm{MeV}$ has not yet been determined. The data presented here is for electrons without correction for the cover plate.

Pulsing first began on one sample on the seventh orbit. The second sample began pulsing on the ninth orbit. The third sample began pulsing on the eleventh orbit. It takes time to get the samples charged up to a near pulsing level.
Figure 2 shows the time history of high energy electron flux during the first 350 orbits. This may be compared to the time history of the pulsing rate to form one of many correlations which are available. The time history of the electron flux from $150 \mathrm{keV}$ to $1.25 \mathrm{MeV}$ has not yet been fully determined so it is not presented. However, the intensity of the electrons in this energy interval usually rises and falls at the same times as does the intensity of the electrons above $1.25 \mathrm{MeV}$.

Figure 3 shows the time history of pulses from the IDM. As time goes on, the pulse rates from some samples are increasing while others are decreasing.

Comparison of figures 2 and 3 provides an important finding which is in agreement with the accumulation of many laboratory experiments [5], but which may contradict an individual experiment. As an overall average, the pulse rate is crudely proportional to the flux of high energy electrons. However, some samples may pulse profusely at the beginning and pulse sparsely after extended radiation time. Other samples may pulse only after extended irradiation or after extended exposure to vacuum. Two small samples cut from a larger piece may show very different pulsing statistics.

It is tempting to deduce a floor for the electron flux which is required to cause pulsing. Figures 2 and 3 would lead one to think that this can be done. We do not yet do this for two good physical reasons.

1 . We do not yet have good data for the flux of electrons from $150 \mathrm{keV}$ to $1.25 \mathrm{MeV}$. This component of the energy distribution is probably important for charging of samples. We are now working on this part of the CRRES data base. It requires that we transport the electrons through the $0.2 \mathrm{~mm}$ aluminum cover shect.

2. The high energy electron fluxes which pass through the samples cause the samples to be more conductive and thereby "leak off charge and lower the peak electric field in the samples." This added conductivity can reduce the probability of pulsing. Using figures 2 and 3 to choose a floor below which one will not have pulsing presumes that this "leak off charge" process is minimized in the IDM samples. No attempt has been made to minimize the leak off process. For example, perhaps a sample mounted on a long narrow insulating post would pulse at much reduced fluxes. Any floor for pulsing deduced from this data would be true only for this set of samples, and can not be extrapolated to other cases. 


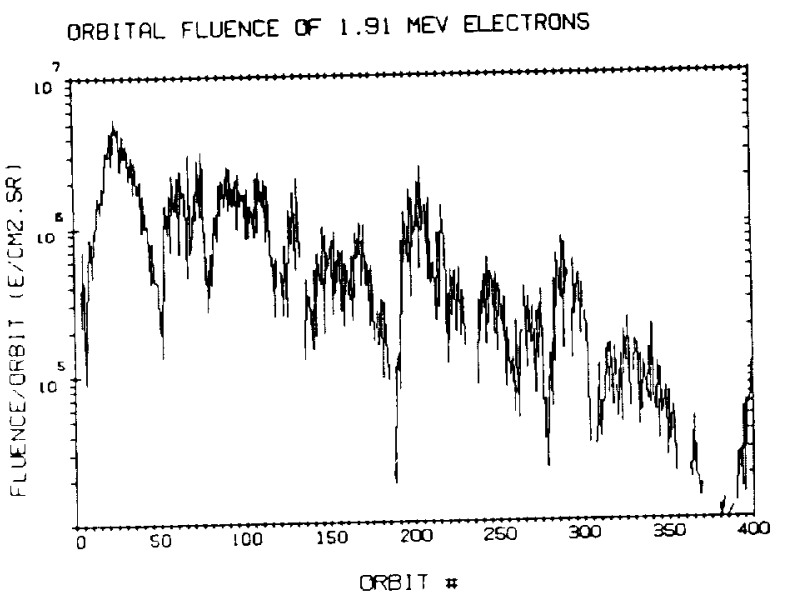

Figure 2. Time History of the Flux Rate From Electrons in a $400 \mathrm{keV}$ Channel at $1.91 \mathrm{MeV}$.

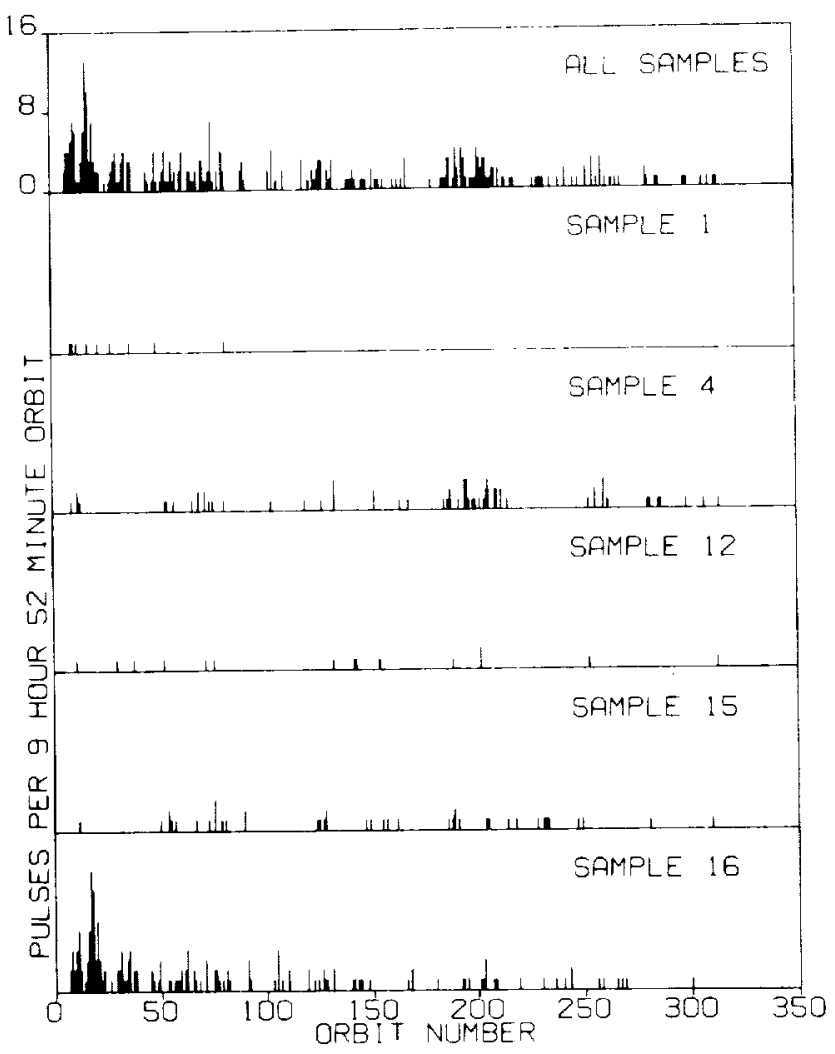

Figure 3. Time History of the Pulse Rates from the IDM Samples. Most of the samples have never pulsed. The sample number corresponds to Table I and is indicated on its graph. These are bar graphs with one bar for each of the orbits. The height of the bar indicates the number of pulses during that orbit.
Nevertheless, we do wish to point out that the samples' pulse rate decays with time much as the electron intensity decays as shown by figures 2 and 3. There is a feeling among some in the spacecraft community that pulsing can be correlated with outgassing from the samples. The IDM results do not show that such a correlation is significant. These samples have been in space a long time. There was a large increase of the electron flux around orbit 600 and the pulse rate responded nearly in proportion. If outgassing is an important parameter, then the IDM samples must have the unusual property of outgassing at a nearly constant rate since the tenth orbit, a period of eight months.

Comparison of figure 3 with Table I puts a stop to some common presumptions that were based upon limited laboratory experiments. For example, one should not predict the rate of discharging from one sample based on the rate experienced from a similar sample; see reference 5 for more on this issue. All the samples in Table I were seen to pulse in laboratory tests [1], but only five have pulsed in space in the first 400 orbits ( 5 months). It is interesting that the samples with configurations 3-8 in Table I have not pulsed. Some have predicted that configurations 3-8 would pulse the most because of the floating metal or other metal edges on the samples. The IDM data indicates that one should not focus on floating metal as an issue of any significance for radiation-induced spacecraft anomalies. For more ideas concerning the variability of pulsing phenomena from apparently similar samples, see references 5-8. The floating metal issue is commented in reference 8 at the bottom of page 228.

The IDM is beginning to accumulate enough data that we may speculate on the causes of the pulsing. Figure 4 is developed to help us do that. In this figure we present the time history of the CRRES IDM during one average orbit which is a composite of the first 350 orbits. We begin the orbit at apogee where the electron flux is low. The curve is a trace of the average electron flux experienced by the IDM during the 350 orbits. The electron flux rises as the satellite moves from apogee at the left end of the graph. On average, the satellite experienced the peak electron fluxes (at an $\mathrm{L}$ shell of 3.5 to 4.5 ) about 13000 seconds after apogee. The electron fluxes diminish around perigee and then peak up again at 24000 seconds after apogee. The satellite attains apogee again at roughly 36000 seconds. 


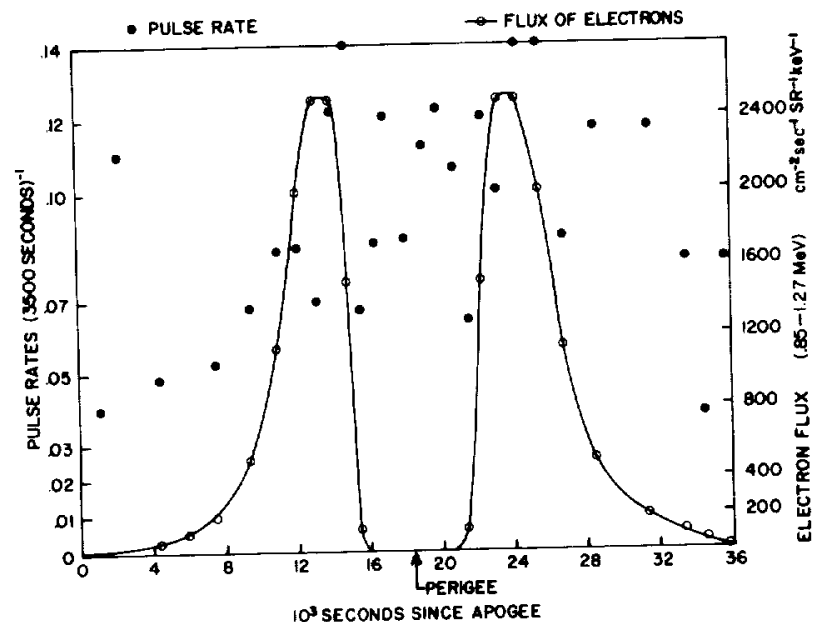

Figure 4. An Average Orbit Time History of the High Energy Electron Flux and IDM Pulse Rates Starting and Ending at Apogee. The solid dots are the measured IDM pulse rates. The flux data is for one electron energy channel, 0.85 to $1.27 \mathrm{MeV}$. Inclusion of all electron energies would not change the shape of this curve in any significant way.

The pulse rate is shown as a histogram on figure 4 . We collected data in $1 / 2 \mathrm{~L}$ shell bins so that there are 28 separate "bins" containing pulse rate data on this graph. Note that CRRES apogee varies, typically from $L=7$ to $L=9$, and everything above $\mathrm{L}=7.5$ is accumulated as one bin; this is a reasonable thing to do because the flux is not contributing much here. Similarly, the data from " $\mathrm{L}=0$ " to $\mathrm{L}=1.5$ is lumped together. With so many bins and only 360 pulses, the statistics of the scatter data is not good.

The pulse rate rises to a maximum after passing through the electron belt on its outward trajectory at around 25000 seconds. Thereafter the pulse rate drops almost in half after the satellite passes through apogee and approaches the electron belt on its inward pass. This decay of pulse rate at high altitudes while near apogee is in reasonable agreement with laboratory experience as reported in reference 5 , page 347 , where samples made with fiberglass pulsed for days after the irradiation ended. Based on figure 4 , very loosly speaking, one could say that the pulse rate, for average IDM pulses, decays in half after about 5 hours without radiation. The time spent at perigee without electron irradiation, being only 2 hours, is not long enough to see any decay in the data.
Figure 4 presents an important fact that must be remembered when interpreting IDM (or other spacecraft anomaly) pulse data. Pulses do not occur because of the instantaneous radiation flux intensity. Pulses result from the accumulation of charge in the insulator which produces high electric fields. The high electric fields, acting alone, do not produce the pulses either. Instead, at the magnitude of electric fields expected [8] in these samples, typical insulators occasionally and spontaneously produce pulses called prebreakdown events. The high voltage insulator literature has much information on this phenomena, yet it cannot be predicted apriori with certainty. The electric fields in these samples decay very slowly so that pulsing can continue for hours or days after radiation ceases. There should be no surprise in this result, those who work with insulators know of the phenomena.

\section{FUTURE WORK}

Having determined that the pulsing samples are responding to an orbit averaged flux, and not to the instantaneous flux of electrons, we can move on to a search for more correlations. We have begun such a search but the work is tedious. We plan to correlate the pulse rate with the hardness of the electron spectrum. There is an intriguing hint in the raw data that one pulse on a sample causes another pulse to be more likely in the immediate future. Leon Levy has noted this in laboratory experiments and Levy and Frederickson have posited an explanation for it (Presented at URSI, Fall 1990). Based on this model one would be tempted to look for enhanced rates of pulsing due to passage through the proton belts, but such correlations would need to be rationalized with the known effects created by the very large range of proton energies in those belts. At the end of March 1991 the electron belts were enhanced a factor of 50 to 100 and produced extreme levels of pulsing on the IDM. The total pulse count more than doubled in a week, and it was the same samples as before. This new data improves our statistics and will make future correlations even more meaningful. 


\section{SUMMARY}

PULSE RATES APPEAR TO SCALE APPROXIMATELY LINEARLY WITH ELECTRON FLUENCE PER ORBIT.

SAMPLES WITH FLOATING METAL ARE RARELY PULSING, THE SIMPLE GEOMETRY IS PULSING THE MOST.

APPROXIMATELY 600 PULSES HAVE BEEN SEEN IN NINE MONTHS SINCE LAUNCH.

TOTAL SAMPLE AREA IS $300 \mathrm{CM}^{2}$. SCALING TO FULL SATELLITE AREA IMPLIES THAT MANY PULSES PER SATELLITE MAY OCCUR.

PULSES OCCUR EVEN HOURS AFTER PASSING THROUGH THE ELECTRON BELTS. PULSE RATES DECAY WITH APPROX 10 HOUR TIME CONSTANT OUTSIDE THE ELECTRON BELTS.

THESE RESULTS ARE FOR SAMPLES WITH NO BIAS. APPLIED BIAS COULD ENHANCE PULSE RATES OR PULSE MAGNITUDES.

THERE SEEMS TO BE NO CORRELATION OF PULSING WITH OUTGASSING.

THERE HAVE BEEN PERIODS OF LOW ELECTRON FLUX WHERE THE PULSE RATE APPROACHES ZERO, ABOUT 1 PULSE PER WEEK. MAXIMUM RATES ARE ROUGHLY 5 PER HOUR.

\section{ACKNOWLEDGEMENT}

A large number of people worked on the IDM program over the years. There is no appropriate way to acknowledge all of their involvements except to say "Thank you all." Dr. Paul Robinson of JPL has been the most active participant, husbanding the instrument through its development phase.

However, we wish to give a special acknowledgement to a dear departed colleague, Mr. William Huber of AFGL who worked many long hours preparing the IDM for flight. Our thoughts are with Bill.

\section{REFERENCES}

1. P. G. Coakley, M. J. Treadaway and P. A. Robinson, Jr., "Low Flux Laboratory Test of The Internal Discharge Monitor (IDM) Experiment Intended for CRRES," IEEE Trans. Nuc. Sci. 32, No. 6, (Dec 85) 4066-72.

2. A. R. Frederickson, et. al., "Leaky Insulating Paint for Preventing Discharge Anomalies on Circuit Boards," IEEE Trans. Nuc. Sci. 36, No. 6, (Dec 89) 2405-10.

3. K. G. Balmain and G. R. Dubois, "Surface Discharges on Teflon, Mylar and Kapton," IEEE Trans. Nuc. Sci. 26, 5146, (1979).

4. A. R. Frederickson, L. Levy and C. L. Enloe, "Radiation-induced Electrical Discharges in Complex Structures," to be published in IEEE Trans. Elec. Insul.

5. A. R. Frederickson, "Electrical Discharge Pulses in Irradiated Solid Dielectrics in Space," IEEE Trans. Elec. Insul. 18, 3, 377-49, (1983).

6. P. Coakley, M. Treadway, N. Wild, and B. Kitterer, "Discharge Characteristics of Dielectric Materials Examined in Mono-, Dual-, and Spectral Energy Electron Charging Environments," in Spacecraft Environmental Interactions Technology 1983, NASA CP-2359 or AFGL TR-85-0018, 51124. (NTIS \#N85-22470)

7. A. R. Frederickson, "Discharge Pulse Phenomenology," ibid, 483-510.

8. A. R. Frederickson, "Partial Discharge Phenomena in Space Applications," in Fourth European Symposium on Spacecraft Materials in Space Environment, 221-31, ONERA-CERT, Toulouse, France, Sept. 1988. 